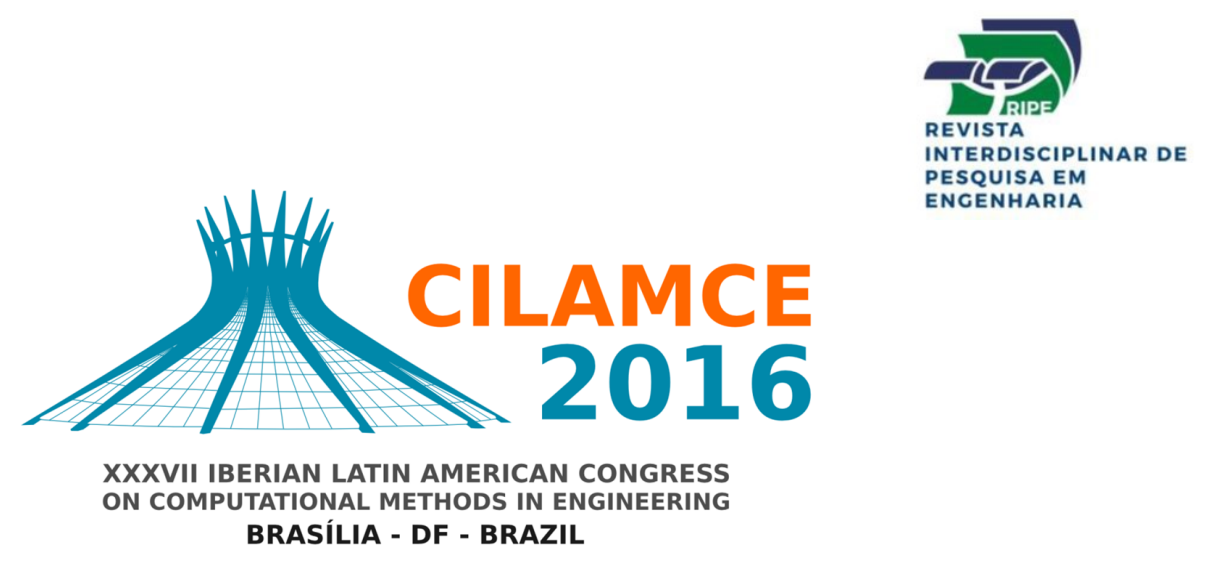

\title{
MECHANICAL ASPECTS OF A GROWING TUMOR
}

\section{Anna Claudia M. Resende}

\section{Regina C. Almeida}

\section{Heber L. Rocha}

annacmr@lncc.br

rcca@lncc.br

heberlr@lncc.br

Laboratório Nacional de Computação Científica (LNCC/MCTI)

Av. Getúlio Vargas 333, 25651-075, Petrópolis, Rio de Janeiro, Brazil

\section{Ernesto A. B. F. Lima}

lima@ices.utexas.edu

Institute for Computational Engineering and Sciences (ICES)

201 East 24th St., 78712-1229, Austin, Texas, USA

Abstract. Cell stresses have a key role on the tumor growth rate, the evolution pattern and on ECM synthesis and organization. In this work, we investigate the interplay between these complex biological phenomena. We develop a heterogeneous tumor growth model subjected to elastic deformation and matrix degradation and remodeling. We incorporate mechanical effects of the surrounding tissue on the growing tumor by assuming that the stress components must satisfy the conservation of linear momentum disregarding inertial effects. We investigate tumor differentiation, morphogenic evolution, and invasion through different feedback mechanism models. Numerical simulations are performed to highlight how mechanical properties of a tissue contribute to cancer progression.

Keywords: Mathematical Model, Tumor Growth, Sensitivity Analysis, Mechanical Properties 


\section{INTRODUCTION}

Tumor environment is composed of different types of cells embedded in the extracellular matrix (ECM), linked by a variety of signal transduction. The cancer progression and invasion is a complex process involving an intricate interplay of a numerous signaling pathways, cellcell and cell-microenvironment mechanical/chemical interactions. In order to grow, the tumor cells need to remodel their environment by interacting with the ECM and promoting the growth of new blood vessels as new source of nutrients. The interactions with the ECM include its degradation in order to facilitate tumor cells mobility and its regeneration in order to serve as a support to cells movement. The degradation and regeneration of the ECM are regulated by a large family of proteolytic enzymes called metalloproteinases (MMPs), whose members express characteristics to either promote or suppress carcinogenesis. MMPs, or more generically, matrix degrading enzymes (MDEs) are secreted by both tumor and normal cells and their functions themselves are balanced by endogenous tissue inhibitors of metalloproteinases. In addition, in order to grow the tumor must overcome the stresses imposed by the surrounding matrix environment as well as the solid stress generated by cellular growth and remodeling. Thus, cell stresses have a key role not only on the tumor growth rate and growth pattern but also on ECM synthesis and organization. It is known that the compressive stress from the environment acting on tumor cells can cause an increase in the apoptosis rate and a decrease in the tumor proliferation (Cheng et al. , 2009; Stylianopoulos et al. , 2012), although the precise feedback mechanism remains obscure. Aiming to include and investigate the interplay for these complex biological phenomena, we develop a reaction-diffusion tumor growth model that it is subjected to elastic deformation and matrix degradation and remodeling due to MMPs.

Beginning with the model developed in Resende (2016), which extends the basic model derived in Hinow et al. (2009); Souza (2013), we first determine the set of influential parameters with respect to the tumor volume evolution. This knowledge makes possible to identify many interesting model properties as well as to how the model might be improved. We then enhance the model by including MDEs, ECM remodeling and mechanical deformation to then evaluate how those new features affect tumor growth evolution.

\section{MATHEMATICAL MODEL}

The baseline model considered here is based on the continuum model developed in references Hinow et al. (2009); Souza (2013), built on mass conservation principles. It consists of seven nonlinear coupled partial differential equations that govern normoxic (proliferative) $(n)$, hypoxic $(h)$ and apoptotic $(a)$ tumor cell densities, extracellular matrix $(f)$ (ECM) and endothelial cells $(m)$ densities, oxygen $(w)$ and vascular endothelial growth factor $(g)$ (VEGF) concentrations. A more complex model derived later refines the ECM sub-model by including matrix degrading enzymes $(p)$ (MDEs) and matrix remodeling. Summarizing, the nondimensional model defined in $\Omega \times\left(0, \tau_{\max }\right]$ is given by the following set of seven coupled partial differential equations: 


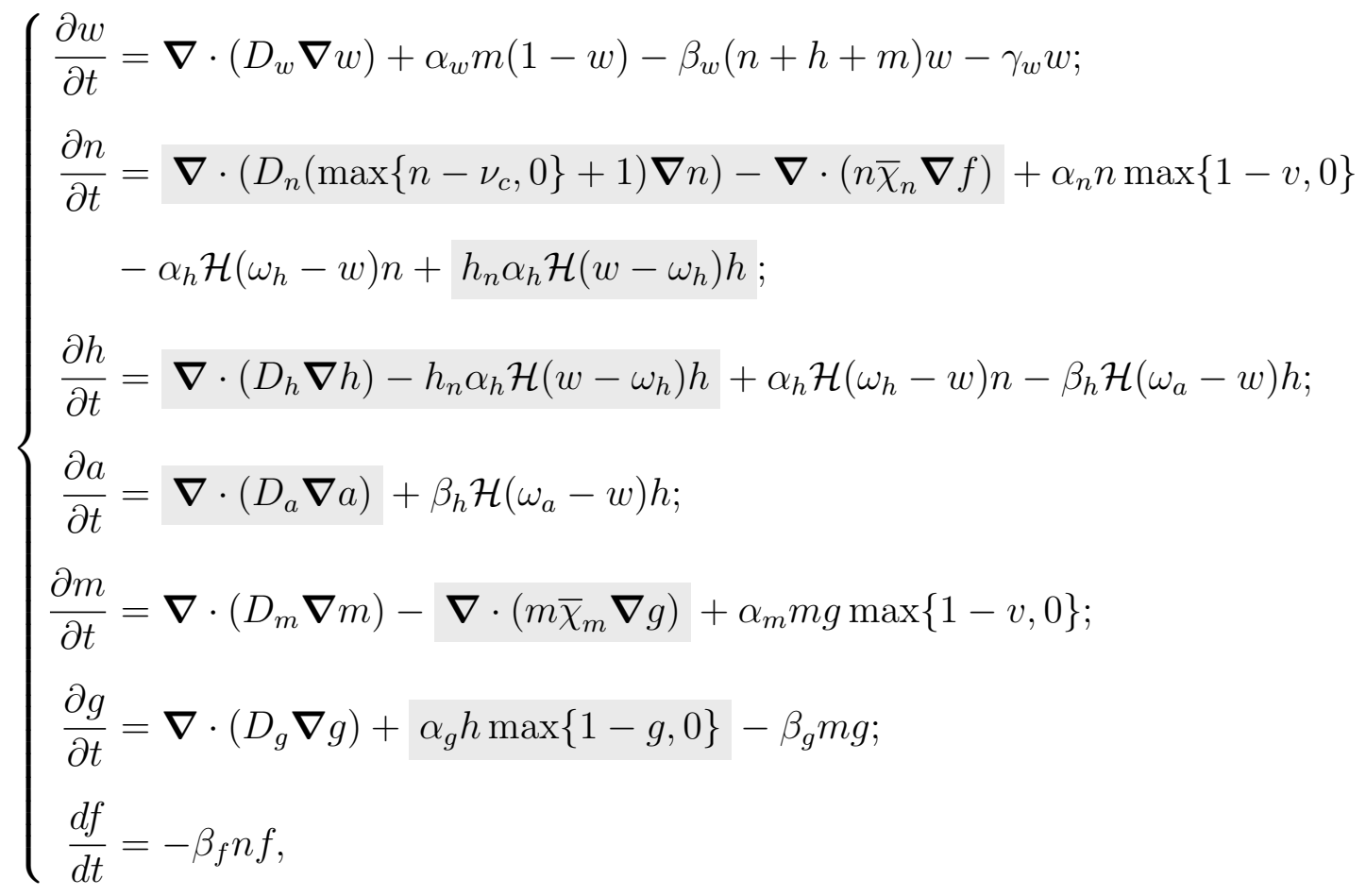

in which $v(\boldsymbol{x}, t)=n(\boldsymbol{x}, t)+h(\boldsymbol{x}, t)+a(\boldsymbol{x}, t)+m(\boldsymbol{x}, t)+f(\boldsymbol{x}, t)$. The highlighted terms indicate modifications we performed in the original model developed in Hinow et al. (2009); Souza (2013). Thus, the baseline model used in our analysis encompasses both avascular and vascular phases of the tumor development. The oxygen concentration is considered the only source of nutrients for cell viability, and $\omega_{h}$ and $\omega_{a}$ represent the oxygen concentrations below which the cell becomes hypoxic and apoptotic, respectively. Phenotypic changes are captured by the Heaviside function $\mathcal{H}$. Tumor-associated angiogenesis is trigged by the VEGF secreted by hypoxic cells, which drives the growth of endothelial cells. Finally, all variables diffuse randomly through the domain, except for the ECM, which is degraded by the normoxic cells invasion. The meaning of all parameters of (1) is described in Table 1.

Table 1: Nondimensional parameter vector $(X)$ for the original one-dimensional model (1).

\begin{tabular}{|l|l|l|l|l|l|}
\hline $\boldsymbol{X}$ & Value & Meaning & $\boldsymbol{X}$ & Value & Meaning \\
\hline$D_{w}$ & 0.58 & nutrient diffusion coeff. & $\beta_{w}$ & 0.57 & oxygen consumption rate \\
\hline$D_{n}$ & $5.8 \times 10^{-5}$ & normoxic cells diffusion coeff. & $\beta_{h}$ & 0.32 & transfer rate from $h$ to $a$ \\
\hline$D_{h}$ & $1.0 \times 10^{-5}$ & hypoxic cells diffusion coeff. & $\beta_{f}$ & 0.5 & rate of ECM degradation \\
\hline$D_{a}$ & $1.0 \times 10^{-6}$ & apoptotic cells diffusion coeff. & $\beta_{g}$ & 5.0 & VEGF consumption rate \\
\hline$D_{m}$ & $5.8 \times 10^{-5}$ & endothelial cells diffusion coeff. & $\omega_{h}$ & 0.4 & threshold $n$ to $h$ \\
\hline$D_{g}$ & 0.02 & VEGF diffusion coeff. & $\omega_{a}$ & 0.3 & threshold $h$ to $a$ \\
\hline$\alpha_{w}$ & 1.0 & rate of oxygen growth & $\chi_{n}$ & $1.4 \times 10^{-4}$ & haptotactic constant $(n)$ \\
\hline$\alpha_{n}$ & $\log 2$ & rate of normoxic cells prolif. & $\chi_{m}$ & $2.1 \times 10^{-6}$ & haptotactic constant $(m)$ \\
\hline$\alpha_{h}$ & 1.6 & transfer rate from $n$ to $h$ & $\gamma_{w}$ & 0.025 & oxygen decay rate \\
\hline$\alpha_{m}$ & 0.7 & rate of endothelial cells growth & $\nu_{c}$ & 0.8 & crowding constant \\
\hline$\alpha_{g}$ & 10.0 & rate of growth of VEGF & $h_{n}$ & 0.1 & $\%$ transfer rate from $h$ to $n$ \\
\hline
\end{tabular}


We develop a stabilized finite element method for solving (1). The details on model solution and numerical methodology are presented in Resende (2016).

\section{SENSITIVITY ANALYSIS}

We now assume that the $d=22$ model parameters are uncertain, each one following a continuous uniform distribution $\mathcal{U}\left(0.8 \bar{X}_{i}, 1.2 \bar{X}_{i}\right)$, in which $\bar{X}_{i}$ is the average value of $X_{i}$ listed in Table 1. We investigate how those uncertainties affect the tumor volume $Y(\boldsymbol{X})$ at given time at which a central core of apoptotic cells is completely developed. For an appropriate set of initial condition and boundary conditions, the sensitivity analysis is performed for a onedimensional problem.

We first use the simple and informative scatterplots method (Saltelli et al. , 2008). We sampled 800 sets of $\boldsymbol{X}$ through a Monte Carlo approach and carried out a SA. The scatterplot patterns shown in Fig. 1 reveal the most important parameters of the model, although do not rank them in the order of importance. This is accomplished by using the elementary effects (EEs) method (Morris, 1991; Saltelli et al. , 2008). EEs is a screening method that provides two SA measures: the average $\left(\mu_{i}^{*}=1 / r \sum_{j=1}^{r}\left|E E_{i}^{j}\right|\right)$ and the standard deviation $\sigma_{i}$ for each $i$ th parameter, that assess its overall importance and nonlinear interactions, respectively. The parametric space is screened through $r$ trajectories of $(d+1)$ points, each one providing $d$ elementary effects for a total of $r(d+1)$ sample points. Beginning with a base vector, randomly sampled in a $p$-level grid, the trajectory points are built by increasing one component by $\Delta$ so that two consecutive points differ in only one component (Saltelli et al. , 2008). Thus, the $i$ th $\mathrm{EE}$ is given by:

$$
E E_{i}=\frac{Y\left(X_{1}, \ldots, X_{i}+\Delta, \ldots, X_{d}\right)-Y(\mathbf{X})}{\Delta}=\frac{Y\left(\mathbf{X}+\mathbf{e}_{i} \Delta\right)-Y(\mathbf{X})}{\Delta} .
$$

The implementation details are described in Resende (2016). Figure 2 shows the global SA measures that allow to identify the set $\left\{D_{n}, \omega_{a}, \beta_{w}, \beta_{f}, \alpha_{n}, D_{w}\right\}$ of the most influential parameters and their order of importance. Remark that the same set of parameters was identified by the scatterplots approach (Fig. 1). In summary, this analysis points out three major features that play crucial roles on the tumor volume evolution:

- Nutrient availability: nutrient uptake and dispersion;

- Tumor cell viability: cell proliferation and dispersion;

- ECM organization.

On the other hand, we may also assume that we might simplify the model by disregarding the non-influential terms characterized by the non-influential parameters pointed out by the SA. They are: (i) the natural oxygen decay; (ii) the increase of the oxygen diffusion coefficient to avoid crowding; (iii) the haptotactic movement of normoxic cells towards ECM's gradient; (iv) the chemotactic movement of endothelial cells towards VEGF's gradient. Unexpectedly, the parameters associated with the angiogenesis were deemed unimportant, which could indicate a major model flaw. This behavior might be due to the fact that there is no explicit interspecific competition between normal and tumor cells, known for playing important role in the angiogenesis process. This issue will be the focus of further investigation. From now, we want to focus on developing a hierarchical family of model based on the information provided by the $\mathrm{SA}$, focusing on model enhancement with respect to tumor proliferation and ECM structure. 

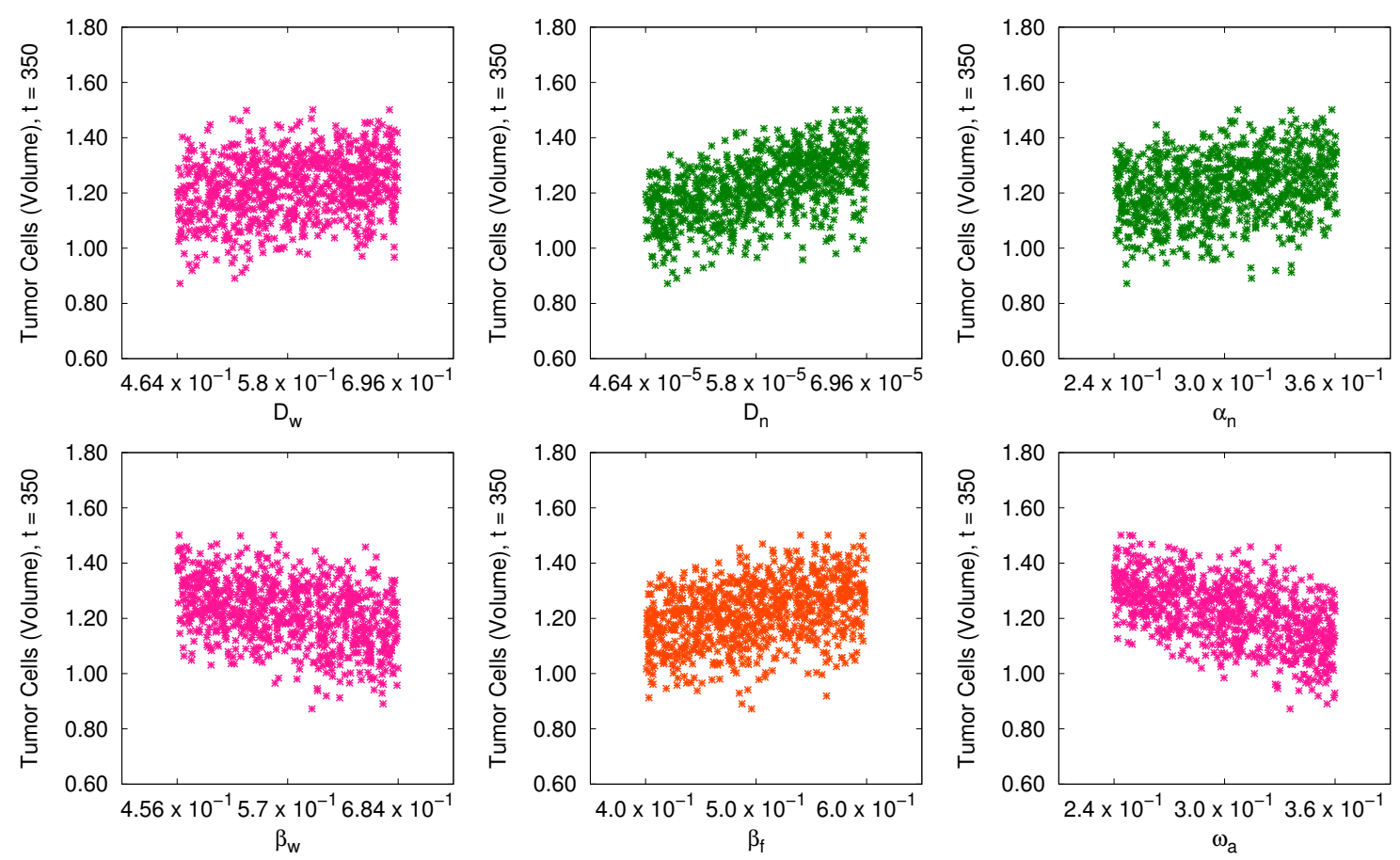

Figure 1: Scatterplots of tumor volume versus the most important parameters of our baseline model (1).
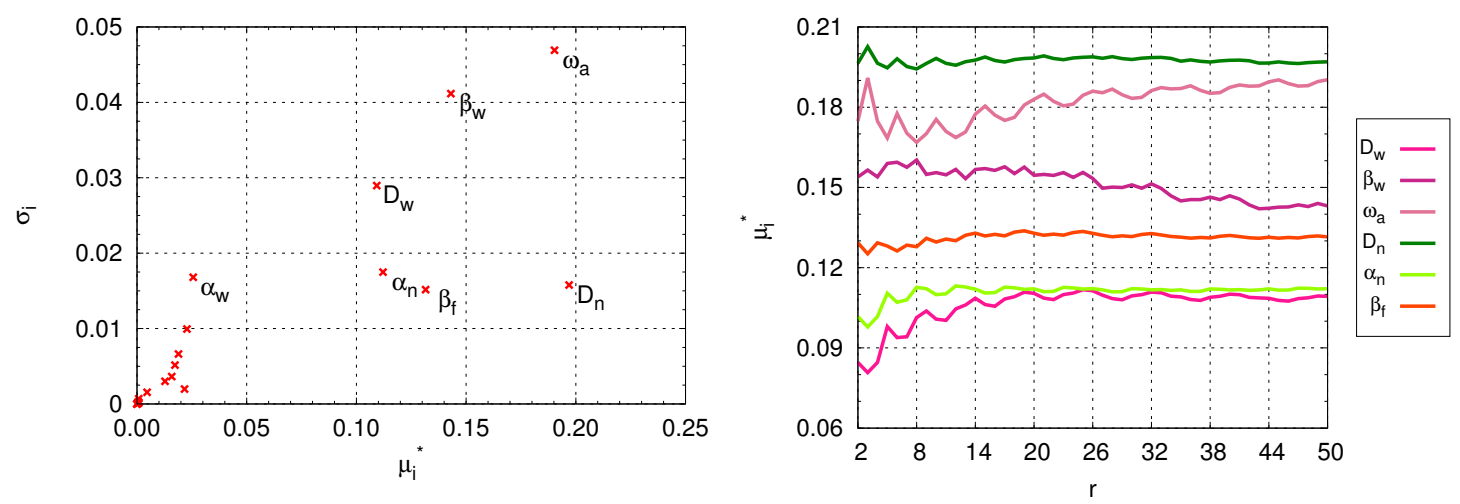

Figure 2: Left: global EEs measures for our baseline model (1) using $p=4$ and $r=50$. Right: $\mu_{i}^{*}$ with respect to the number of trajectories $r$, with higher $\mu_{i}^{*}$ indicating a more important parameter.

\section{HIERARCHICAL FAMILY OF TUMOR GROWTH MODELS}

We expect to guarantee that all models in the family have at least the same set of important parameters of the original model. In this way, the hierarchical family of models satisfies the following hypotheses:

(a) The medium is heterogeneous;

(b) Angiogenesis is triggered during the tumor evolution thus implying that tumor cells undergo different phenotypic stages;

(c) Normoxic cells proliferate at rate $\alpha_{n}$;

(d) The transitions between cell stages mainly depend on the threshold $\omega_{a}$ (and so on $\omega_{h}$ ); 
(e) The oxygen transport depends on the diffusion coefficient $D_{w}$ and oxygen uptake rate $\beta_{w}$;

(f) Tumor evolution depends on the extracellular matrix degradation $\left(\beta_{f}\right)$ and on normoxic cells motility $\left(D_{n}\right)$.

Let us now consider two new models of the family. The first one is obtained by disregarding some of the non-influential phenomena pointed out by the SA, i.e., dropping the following terms of (1): (i) the natural oxygen decay; (ii) the increase of the oxygen diffusion coefficient to avoid crowding; (iii) the haptotactic movement of normoxic cells towards ECM's gradient; (iv) the chemotactic movement of endothelial cells towards VEGF's gradient. It is worth mentioning that some terms related to parameters not identified as important by the SA, as $\alpha_{w}$ and $h_{n}$, for example, are kept due to the general issues defined previously. More specifically, $\alpha_{w}$ and $h_{n}$ play important roles in angiogenesis and on the transitions between cell stages, respectively, so that they can not be disregarded. This new model is mathematically described by the following system of equations:

$$
\left\{\begin{array}{l}
\frac{\partial w}{\partial t}=\boldsymbol{\nabla} \cdot\left(D_{w} \nabla w\right)+\alpha_{w} m(1-w)-\beta_{w}(n+h+m) w \\
\frac{\partial n}{\partial t}=\boldsymbol{\nabla} \cdot\left(D_{n} \boldsymbol{\nabla} n\right)+\alpha_{n} n \max \{1-v, 0\}-\alpha_{h} \mathcal{H}\left(\omega_{h}-w\right) n+h_{n} \alpha_{h} \mathcal{H}\left(w-\omega_{h}\right) h \\
\frac{\partial h}{\partial t}=\nabla \cdot\left(D_{h} \nabla h\right)-h_{n} \alpha_{h} \mathcal{H}\left(w-\omega_{h}\right) h+\alpha_{h} \mathcal{H}\left(\omega_{h}-w\right) n-\beta_{h} \mathcal{H}\left(\omega_{a}-w\right) h \\
\frac{\partial a}{\partial t}=\boldsymbol{\nabla} \cdot\left(D_{a} \boldsymbol{\nabla} a\right)+\beta_{h} \mathcal{H}\left(\omega_{a}-w\right) h \\
\frac{\partial m}{\partial t}=\boldsymbol{\nabla} \cdot\left(D_{m} \boldsymbol{\nabla} m\right)+\alpha_{m} m g \max \{1-v, 0\} \\
\frac{\partial g}{\partial t}=\boldsymbol{\nabla} \cdot\left(D_{g} \boldsymbol{\nabla} g\right)+\alpha_{g} h \max \{1-g, 0\}-\beta_{g} m g \\
\frac{d f}{d t}=-\beta_{f} n f .
\end{array}\right.
$$

The sensitivity analysis showed that ECM degradation plays an important role on tumor evolution. Actually, tumor invasion is a process that involves degradation of a preexisting local connective tissue (Madsen \& Bugge, 2015). Recent studies have shown that some extracellular matrix degrading enzymes (MDEs), such as matrix metalloproteinases (MMPs), are key regulators on cancer development and progression. Thus, we enhance the ECM's sub-model by considering the role of MMPs. We assume that MDEs dynamics are soluble so that they are transported in the domain through diffusion. MDEs present a natural decay and we consider that they are produced by normoxic cells up to a maximum concentration $p_{\max }$ in the microenvironment (Oden et al. , 2015). MDEs produced by normoxic cells are now responsible for ECM degradation. Another new feature of the enhanced model is the ECM remodeling by tumor cells if there is enough space in the microenvironment. Thus, this new model is described by the following system of equations: 


$$
\left\{\begin{array}{l}
\frac{\partial w}{\partial t}=\nabla \cdot\left(D_{w} \nabla w\right)+\alpha_{w} m(1-w)-\beta_{w}(n+h+m) w \\
\frac{\partial n}{\partial t}=\nabla \cdot\left(D_{n} \nabla n\right)+\alpha_{n} n \max \{1-v, 0\}-\alpha_{h} \mathcal{H}\left(\omega_{h}-w\right) n+h_{n} \alpha_{h} \mathcal{H}\left(w-\omega_{h}\right) h \\
\frac{\partial h}{\partial t}=\nabla \cdot\left(D_{h} \nabla h\right)-h_{n} \alpha_{h} \mathcal{H}\left(w-\omega_{h}\right) h+\alpha_{h} \mathcal{H}\left(\omega_{h}-w\right) n-\beta_{h} \mathcal{H}\left(\omega_{a}-w\right) h \\
\frac{\partial a}{\partial t}=\nabla \cdot\left(D_{a} \nabla a\right)+\beta_{h} \mathcal{H}\left(\omega_{a}-w\right) h \\
\frac{\partial m}{\partial t}=\nabla \cdot\left(D_{m} \nabla m\right)+\alpha_{m} m g \max \{1-v, 0\} \\
\frac{\partial g}{\partial t}=\nabla \cdot\left(D_{g} \nabla g\right)+\alpha_{g} h \max \{1-g, 0\}-\beta_{g} m g \\
\frac{\partial p}{\partial t}=\nabla \cdot\left(D_{p} \nabla p\right)+\alpha_{p} n(1-p)-\beta_{p} p-\beta_{f} p f \\
\frac{d f}{d t}=-\beta_{f} p f+\alpha_{f} n \max \{1-v, 0\} .
\end{array}\right.
$$

The new parameters are listed in Table 2. As indicated in Resende (2016), the SA performed to these two new models indicate that the same set of important parameters remains indeed the same. We emphasize that the modeling framework in this manner provides a powerful way for studying a model itself or either its simplification or extension. The framework can also be tailored to form the basis for future models, incorporating new processes and phenomena.

Table 2: Nondimensional parameters for the enhanced one-dimensional model (1).

\begin{tabular}{|l|l|l|}
\hline Parameter & Value & Meaning \\
\hline$D_{p}$ & 0.1 & MDE diffusion coefficient \\
\hline$\alpha_{p}$ & 7.0 & rate of MDE production by proliferative cells \\
\hline$\alpha_{f}$ & 0.3 & rate of ECM regeneration \\
\hline$\beta_{p}$ & 10.0 & MDE natural decay \\
\hline$\beta_{f}$ & 1.0 & rate of ECM degradation \\
\hline \hline
\end{tabular}

\section{CANCER MECHANICS}

Tumor micro-environment is highly heterogeneous, composed of different types of cells embedded in the ECM. In order to grow, cancer cells must overcome the stresses imposed by the surrounding matrix environment as well as the solid stress generated by cellular growth and remodeling. To define the deformation model we first introduce the displacement field, denoted by $\boldsymbol{u}$, and we assume a small infinitesimal displacement regime. This implies that the deformation (strain) tensor is given by:

$$
\boldsymbol{\varepsilon}=\frac{1}{2}\left(\boldsymbol{\nabla} \boldsymbol{u}+\boldsymbol{\nabla} \boldsymbol{u}^{t}\right)
$$


which may be split into the form:

$$
\varepsilon=\varepsilon^{s}+\varepsilon^{g}
$$

Here, the mechanical counterpart of strain due to applied stress is denoted by $\varepsilon^{s}$ and $\varepsilon^{g}$ stands for the stress-free strain due to growth. Denoting $\phi=n+h+a$ as the total tumor cell density, we define the linear elastic inhomogeneous material tensor as:

$$
\mathcal{C}(\phi)=\mathcal{C}^{h}+g(\phi)\left(\mathcal{C}^{t}-\mathcal{C}^{h}\right)
$$

The function $g(\phi)$ is an interpolation function that allows $\mathcal{C}(\phi)$ to vary smoothly across the interface from the healthy tissue $\left(\mathcal{C}^{h}\right)$ to the tumor tissue $\left(\mathcal{C}^{t}\right)$. To this end, $g(\phi)$ satisfies $g(\phi=1)=1$ in regions where there are only tumor cells, and $g(\phi=0)=0$ elsewhere (Tsukada et al. , 2009; Wise et al. , 2008). The strain energy density may now be defined by:

$$
W=\frac{1}{2} \varepsilon: \mathcal{C}(\phi) \varepsilon+\varepsilon: \bar{\sigma}(\phi)
$$

in which $\bar{\sigma}(\phi)$ is a symmetric compositional stress tensor associated with the tumor growth. Assuming that the diffusion mechanism occurs on a time scale much larger than that associated with inertia, we may consider a quasi-static approximation for the linear momentum balance. Using Eq. (8) and assuming no body forces and no additional momentum supplied, the equilibrium equation is simply given by:

$$
\boldsymbol{\nabla} \cdot(\mathcal{C}(\phi) \boldsymbol{\varepsilon}+\bar{\sigma}(\phi))=0
$$

For this preliminary study on the tumor mechanical effects, we assume a linear isotropic compositional stress of the form:

$$
\bar{\sigma}(\phi)=\lambda n \mathcal{I},
$$

in which $\mathcal{I}$ is the identity tensor and $\lambda$ depends on the tumor proliferative growth rate. Thus, rewriting Eq. (9) in terms of the displacement vector $\boldsymbol{u}$, we obtain:

$$
\frac{1}{2} \boldsymbol{\nabla} \cdot\left[\mathcal{C}(\phi)\left(\nabla \boldsymbol{u}+\nabla \boldsymbol{u}^{t}\right)\right]=-\lambda \nabla n
$$

A noteworthy simplification happens by assuming that the tumor microenvironment is isotropic and homogeneous so that Hooke's law holds (Weis et al. , 2013). Defining the Poisson's ratio $\nu$, the Young's modulus $E$, and the shear modulus $G=E /(2(1+\nu))$, the equilibrium may be written as:

$$
0=\boldsymbol{\nabla} \cdot(\mathrm{G} \boldsymbol{\nabla} \boldsymbol{u})+\boldsymbol{\nabla} \frac{\mathrm{G}}{1-2 \nu}(\boldsymbol{\nabla} \cdot \boldsymbol{u})+\lambda \boldsymbol{\nabla} n .
$$

The progressive accumulation of compressive stresses inside the tumor as it grows has a huge impact on cell behavior and on the whole microenvironment. According to the experimental studies performed by Cheng et al. (2009); Stylianopoulos et al. (2012), the accumulation of 
compressive stresses inside tumors inhibits tumor growth and changes the tumor growth pattern. Here, we investigate the effects of compressive stresses on the tumor proliferative growth rate and normoxic cell diffusion coefficient by introducing a negative feedback control following the work developed by Weis et al., 2013. By defining a scaling constant $\gamma$ and the Von Mises stress $\sigma_{\mathrm{vm}}$, the control mechanism is modeled by an exponential damping factor $\mathcal{D}^{f a c}$ given by:

$$
\mathcal{D}^{f a c}=e^{-\gamma \sigma_{\mathrm{vm}}} .
$$

This damping factor modulates either the tumor proliferative growth rate or the normoxic cell diffusion coefficient such that they become the heterogeneous parameters given by:

$$
\bar{D}_{n}=\mathcal{D}^{f a c} D_{n}=D_{n} e^{-\gamma \sigma_{\mathrm{vm}}} \quad \text { and } \quad \bar{\alpha}_{n}=\mathcal{D}^{f a c} \alpha_{n}=\alpha_{n} e^{-\gamma \sigma_{\mathrm{vm}}} .
$$

In the following, we solve the 2D coupled model under the previous equilibrium approximation in order to investigate tumor differentiation, morphogenic evolution, and invasion through the two different feedback mechanism models given by Eq. (14) using the parameters defined in Table 3.

Table 3: Parameters for the simulation of the two-dimensional model (1) with mechanical coupling.

\begin{tabular}{|c|c|c|}
\hline \hline Parameter & Value & Meaning \\
\hline$E$ & $2.0 \mathrm{kPa}$ & Young's Modulus \\
\hline$G$ & $0.69 \mathrm{kPa}$ & Shear Modulus \\
\hline$\nu$ & 0.45 & Poisson's Rate \\
\hline$\gamma$ & $2.0 \times 10^{-5} \mathrm{kPa}^{-1}$ & Mechanical Coupling Coefficient \\
\hline$\lambda$ & $2.0 \times 10^{-5}$ & Scaling Constant \\
\hline
\end{tabular}

\section{NUMERICAL SIMULATION}

In the preliminary simulations that we present here, we consider the most complete model of the hierarchical family described previously, with and without considering mechanical deformation. We consider a two-dimensional computational domain $\Omega=(0,3) \times(0,3)$ in which the nutrient is assumed to be $w(\boldsymbol{x}, 0)=1.0$. The initial condition for normoxic cells is assumed to be of the following circular shape:

$$
\left\{(x, y): \frac{(x-1.5)^{2}}{0.01}+\frac{(y-1.5)^{2}}{0.01} \leq 1.0\right\} \text {. }
$$

We assume that there is no hypoxic cells initially as well as VEGF concentration, and basal values for apoptotic and endothelial cells are stated over the domain $\Omega: a(\boldsymbol{x}, 0)=5 \times 10^{-2}$; $m(\boldsymbol{x}, 0)=1 \times 10^{-2}$. Thus, the ECM density in the microenvironment is given by:

$$
\begin{aligned}
f(\boldsymbol{x}, 0) & =1.0-n(\boldsymbol{x}, 0)-h(\boldsymbol{x}, 0)-a(\boldsymbol{x}, 0)-m(\boldsymbol{x}, 0) ; \\
& =1.0-n(\boldsymbol{x}, 0)-0.06 .
\end{aligned}
$$

The dynamics is completely described by defining the following Dirichlet and homogeneous Neumann conditions:

$$
\left.\begin{array}{r}
\nabla n \cdot \boldsymbol{n}=\boldsymbol{\nabla} h \cdot \boldsymbol{n}=\boldsymbol{\nabla} a \cdot \boldsymbol{n}=0 \\
\nabla m \cdot \boldsymbol{n}=\boldsymbol{\nabla} f \cdot \boldsymbol{n}=\boldsymbol{\nabla} g \cdot \boldsymbol{n}=0 ; \\
w(\boldsymbol{x}, t)=1 ;
\end{array}\right\} \text { on } \Gamma \times\left(0, \tau_{\max }\right]
$$


where $\boldsymbol{n}$ is a unit exterior normal vector on $\Gamma$. These conditions indicate that a constant nutrient supply is stated on the boundary of the domain and no mass flux is allowed. For spatial and temporal accuracy, we use a quadrilateral mesh with $16 \times 10^{4}$ uniform elements $(400 \times 400)$ and a constant time step size $(\Delta t=0.1)$. As these $2 \mathrm{D}$ numerical experiments demand high computational power, we use the $\mathrm{C}++\mathrm{l}$ ibMesh library to take advantage of parallel features.

Figure 3 show the tumor evolution at $t=350$ without the mechanical deformation feedback on the tumor growth. As normoxic cells grow in size and ECM is degraded and remodeled, the oxygen concentration gradually decreases towards the center of the tumor. The onset of hypoxic cells takes place when $w<\omega_{h}$, promoting the growth of a new vascular network around the proliferative rim. Even with the appearance of endothelial cells, the nutrient supply remains insufficient to maintain quiescent tumor cells in the middle of the tumor, yielding the onset of apoptotic cells in this region of the microenvironment, where oxygen concentration drops below $\omega_{a}$.

w

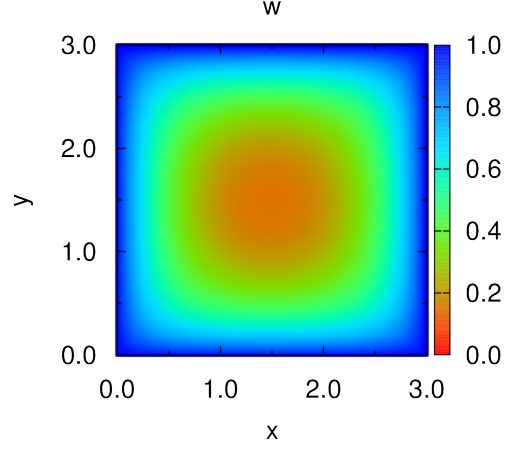

a

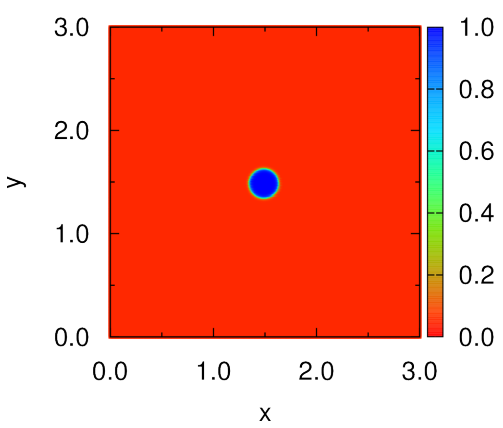

$\mathrm{n}$

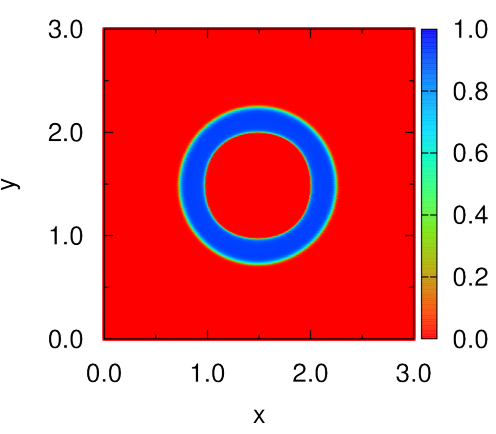

$\mathrm{m}$

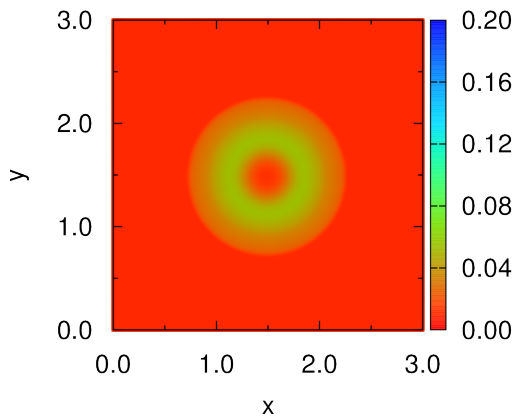

g

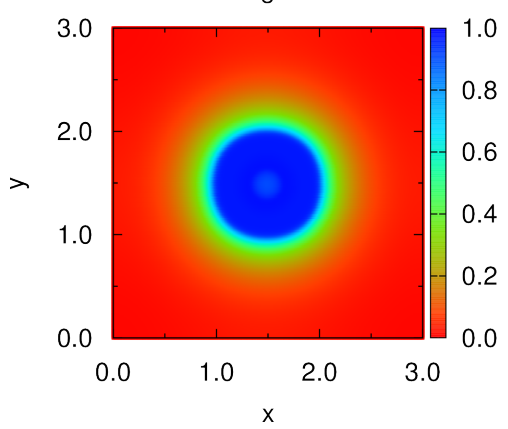

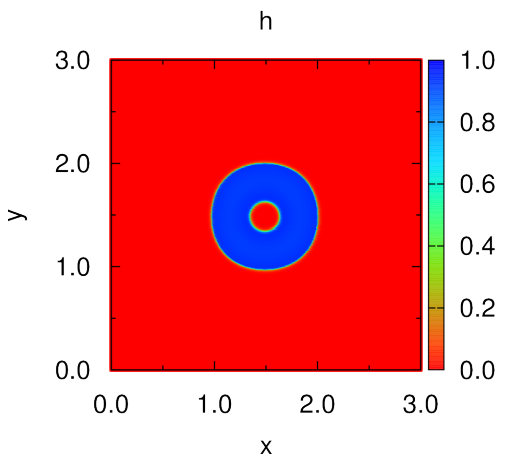

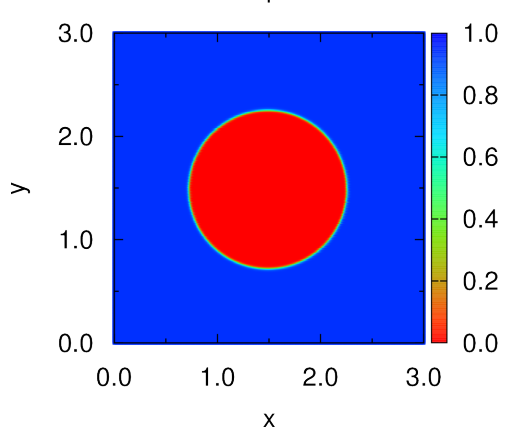

Figure 3: Two-dimensional experiment (original model) at $t=350$. 
We perform the same experiment including the mechanical deformation feedback on the diffusion coefficient. Because of the choices used for $\lambda$ and $\gamma$, the snapshots of the variables evolution at $t=350$ are almost indistinguishable from those depicted in Fig. 3. Figure 4 shows the evolution of the $x$-component of the displacement field at $y=0$. The results are similar to those presented in Garg \& Miga (2008) at early times. As time evolves, we notice a displacement pattern disruption caused by the onset of hypoxic cells. This is explained by the fact that in the present model tumor cells are differentiated into proliferative, hypoxic and apoptotic, and only normoxic cells induce growth.

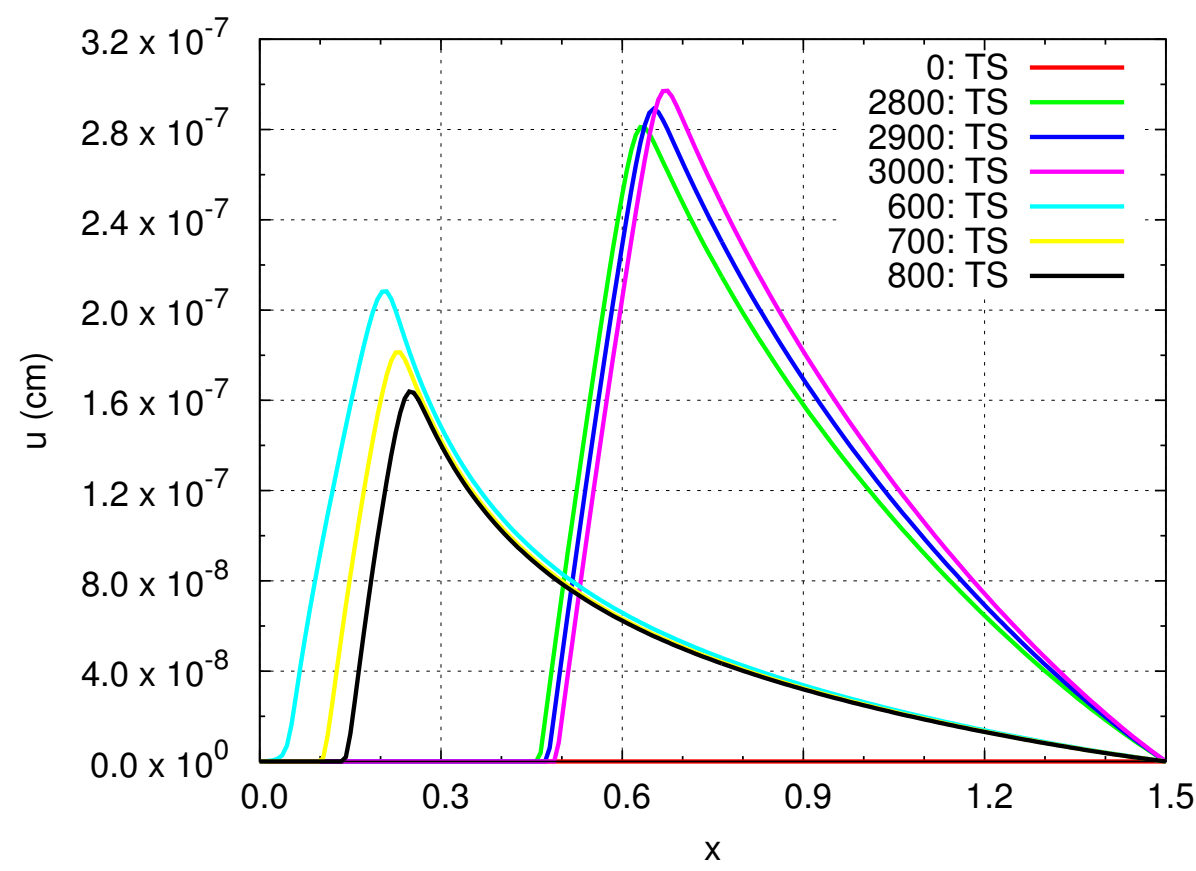

Figure 4: The $x$-component of the displacement field $u$ for different time steps.

\section{CONCLUSION}

In this work we focus on the development of a family of hierarchical deterministic tumor growth model capable of capturing both avascular and vascular phases of cancer as well as the mechanical effects promoted by the tumor growth. Beginning with a baseline vascular model, the impact of parameter uncertainty is assessed by performing SAs through scatterplots and elementary effects techniques. Both were able to identify the set of the most influential parameters with respect to the evolution of the tumor volume. We showed that our overarching hypothesis that a model-building framework may be systematically developed by defining essential model hypotheses and a set of influential parameters, is indeed valid. Some preliminary experiments have showed the interplay between compressive stresses accumulation and the feedback control on the diffusion coefficient. More experiments are needed to verify the ranges of scale parameters in the deformation model as well as to compare the two different feedback models.

\section{ACKNOWLEDGEMENTS}

The authors would like to thank the support through the following projects of CNPq: 132591/2014-6 and 305612/2013-1. 


\section{REFERENCES}

Cheng, G., Tse, J., Jain, R. K., \& Munn, L. L. 2009. Micro-Environmental Mechanical Stress Controls Tumor Spheroid Size and Morphology by Suppressing Proliferation and Inducing Apoptosis in Cancer Cells. PLoS ONE, 4(2), e4632.

Garg, Ishita, \& Miga, Michael I. 2008. Preliminary investigation of the inhibitory effects of mechanical stress in tumor growth. Proc. of SPIE, 6918, 69182L.

Hinow, P., Gerlee, P., McCawley, L. J., et al. . 2009. A spatial model of tumor-host interaction: application of chemotherapy. Mathematical Biosciences and Engineering, 6(3), 521-546.

Madsen, D. H., \& Bugge, T. H. 2015. The source of matrix-degrading enzymes in human cancer: Problems of research reproducibility and possible solutions. The Journal of Cell Biology, 209(2), 195-198.

Morris, M. D. 1991. Factorial Sampling Plans for Preliminary Computational Experiments. Technometrics, 33(2), 161-174.

Oden, J. T., Lima, E. A. B. F., Almeida, R. C., et al. . 2015. Toward Predictive Multiscale Modeling of Vascular Tumor Growth. Archives of Computational Methods in Engineering, $1-45$.

Resende, A. C. M. 2016. Sensitivity Analysis as a Tool for Tumor Growth Modeling. M.Phil. thesis, Laboratório Nacional de Computação Científica (LNCC/MCTI), Petrópolis/RJ, Brazil.

Saltelli, A., Ratto, M., Andres, T., Campolongo, F., Cariboni, J., Gatelli, D., Saisana, M., \& Tarantola, S. 2008. Global Sensitivity Analysis: The Primer. John Wiley \& Sons.

Souza, R. V. M. 2013. Modelos de crescimento tumoral espacialmente heterogêneos com aplicação de quimioterapia. M.Phil. thesis, Laboratório Nacional de Computação Científica (LNCC/MCTI), Petrópolis/RJ, Brazil.

Stylianopoulos, T., Martin, J. D., Chauhan, V. P., Jain, S. R., Diop-Frimpong, B., Bardeesy, N., Smith, B. L., Ferrone, C. R., Hornicek, F. J., Boucher, Y., Munn, L. L., \& Jain, R. K. 2012. Causes, consequences, and remedies for growth-induced solid stress in murine and human tumors. Proceedings of the National Academy of Sciences of the United States of America, 109(38), $15101-15108$.

Tsukada, Yuhki, Murata, Yoshinori, Koyama, Toshiyuki, \& Morinaga, Masahiko. 2009. PhaseField Simulation of the Effect of Elastic Inhomogeneity on Microstructure Evolution in NiBased Superalloys. MATERIALS TRANSACTIONS, 50(4), 744-748.

Weis, J. A., Miga, M. I., Arlinghaus, L. R., Li, X., Chakravarthy, A. B., Abramson, V., Farley, J., \& Yankeelov, T. E. 2013. A mechanically coupled reaction-diffusion model for predicting the response of breast tumors to neoadjuvant chemotherapy. Physics in medicine and biology, 58(17), 5851.

Wise, S. M., Lowengrub, J. S., Frieboes, H.B., \& Cristini, V. 2008. Three-dimensional multispecies nonlinear tumor growth - I Model and numerical method. Journal of Theoretical Biology, 253, 524- 543. 